\title{
Semantic Type Qualifiers
}

\author{
Brian Chin Shane Markstrum Todd Millstein \\ University of California, Los Angeles \\ \{naerbnic,smarkstr,todd\}@cs.ucla.edu
}

\begin{abstract}
We present a new approach for supporting user-defined type refinements, which augment existing types to specify and check additional invariants of interest to programmers. We provide an expressive language in which users define new refinements and associated type rules. These rules are automatically incorporated by an extensible typechecker during static typechecking of programs. Separately, a soundness checker automatically proves that each refinement's type rules ensure the intended invariant, for all possible programs. We have formalized our approach and have instantiated it as a framework for adding new type qualifiers to $\mathrm{C}$ programs. We have used this framework to define and automatically prove sound a host of type qualifiers of different sorts, including pos and neg for integers, tainted and untainted for strings, and nonnull and unique for pointers, and we have applied our qualifiers to ensure important invariants on open-source $C$ programs.
\end{abstract}

Categories and Subject Descriptors D.2.4 [Software/Program Verification]: reliability, validation; D.3.m [Miscellaneous]; F.3.1 [Specifying and Verifying and Reasoning about Programs]: invariants, mechanical verification, specification techniques

General Terms Languages, Reliability, Verification

Keywords type qualifiers, extensible typechecking, type soundness

\section{Introduction}

Type systems are a natural discipline for ensuring that programs maintain certain run-time invariants. As a simple example, if an expression can be given the type int, the programmer is assured (modulo type-unsafe features like casts) that the expression will only ever evaluate dynamically to an integer value. Recent work in our community has shown how to refine the types in traditional type systems to ensure other important kinds of run-time invariants, including memory safety (e.g., [38, 33, 23]), invariants about pointers and their aliasing relationships (e.g., $[8,5,1,15]$ ), and invariants about the interactions of threads in concurrent programs (e.g., $[16,4,18])$. The refinements are achieved by augmenting standard types with annotations that represent the additional properties of interest and augmenting standard type rules to check these annotations statically.

Permission to make digital or hard copies of all or part of this work for personal or classroom use is granted without fee provided that copies are not made or distributed for profi t or commercial advantage and that copies bear this notice and the full citation on the first page. To copy otherwise, to republish, to post on servers or to redistribute to lists, requires prior specifi c permission and/or a fee.

PLDI'05, June 12-15, 2005, Chicago, Illinois, USA.

Copyright (c) 2005 ACM 1-59593-080-9/05/0006... \$5.00.
Of course, language designers cannot anticipate all the runtime invariants that programmers will want to specify and check. Language designers also cannot anticipate all of the practical ways in which types may be refined in order to enforce a particular invariant. Therefore, it is desirable to provide a framework for user-defined type refinements, whereby programmers can easily augment a language's type system with new type annotations to ensure invariants of interest. For example, Foster et al. describe CQUAL $[19,20]$, a system that allows programmers to define new type qualifiers, such as nonnull and untainted, for C programs, and Mandelbaum et al. [27] provide a theory of type refinements to specify and check properties, like temporal protocols, that depend on "effectful" computations.

Despite their benefits, existing frameworks for user-defined type refinements have important limitations that hinder their utility. First, they use a fixed set of type rules across all type refinements. Type-refinement-specific information is provided by annotating each program to indicate when the typechecker should assume or check that a refinement holds on some program fragment. While such annotations can simulate a limited form of refinementspecific type rules, they are not expressive enough to handle many common situations. For example, it would be difficult to simulate a type rule for some expression that depends recursively on the types of subexpressions.

Second, while existing frameworks ensure that programs respect user-defined typing disciplines, the type-refinement designer must take responsibility for ensuring that his new typing discipline in fact guarantees the desired run-time program invariants. Any errors in the user-defined typing discipline are undetected by the framework. For example, a program that is improperly annotated with nonnull assumptions can typecheck in CQUAL but nonetheless cause a variable declared nonnull to have the value NULL at run time.

In this paper, we address these limitations of prior frameworks via a novel approach to user-defined type refinements:

- A language for type-refinement rules. We provide an explicit language in which users write type rules for their new refinements. This language enables the natural expression of userdefined typing disciplines, and it is much more expressive than the program annotations used by prior frameworks. An extensible typechecker automatically incorporates user-defined type rules during static typechecking of programs, in order to enforce users' typing disciplines.

- Semantic guarantees. We allow users to explicitly specify the run-time invariant that a type refinement is meant to represent. We observe that for many interesting cases such invariants are quite natural and simple. A soundness checker automatically proves that each refinement's type rules ensure the intended invariant, for all possible programs.

We have instantiated our approach as a framework for adding user-defined type qualifiers to $\mathrm{C}$ programs. The extensible type- 


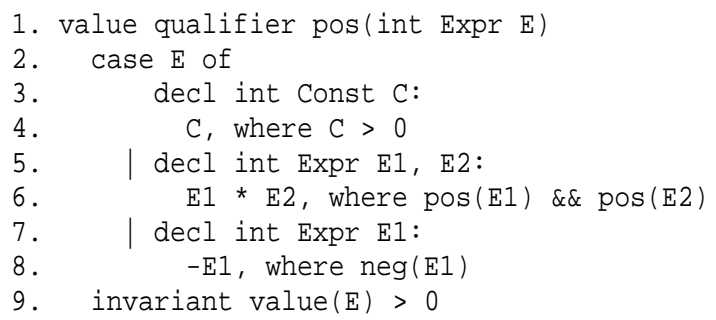

Figure 1. A user-defined type qualifier and associated type rules for positive integers.

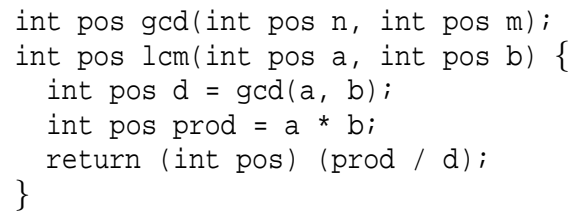

Figure 2. Example code using the pos type qualifier.

checker is implemented in the CIL [32] front end for C. The soundness checker implements a proof strategy that requires a handful of proof obligations to be discharged. Our implementation employs Simplify [14], the automatic theorem prover from the Extended Static Checker for Java (ESC/Java) [17], to automatically discharge these proof obligations. We have used our framework to define and automatically prove sound a host of type qualifiers: qualifiers that restrict the value of an expression, including nonnull, nonzero, pos, and neg; qualifiers that restrict the flow of values through a program, including tainted and untainted [36]; and qualifiers that restrict a program's aliasing relationships, including unique and unaliased. We have used our qualifiers to ensure important program invariants on open-source $\mathrm{C}$ programs.

In the next section, we overview our framework for user-defined type qualifiers informally via a number of examples. Sections 3 and 4 describe the implementations of our extensible typechecker and soundness checker, respectively. Section 5 summarizes the formal details of our approach. Section 6 describes experience using our framework to check $\mathrm{C}$ programs. Section 7 compares with related work, and section 8 concludes and discusses future directions.

\section{Semantic Type Qualifiers}

Our framework supports two common classes of qualifiers, which are discussed in turn below. Value qualifiers, such as pos and nonnull, pertain only to the value of an expression. Reference qualifiers, such as unique and unaliased, (additionally) pertain to the address of an l-valuable expression (or l-value). Distinguishing between these classes of qualifiers allows us to provide specialized support for each one, making it easier for users to define qualifiers and for our framework to reason about them automatically.

\subsection{Value Qualifiers}

Figure 1 illustrates a definition of the value qualifier pos in our framework, which can be used to statically track positive integers. Line 1 of the figure declares pos to be a new value qualifier applicable to expressions of type int. It also declares a variable $\mathrm{E}$, which is used in the rest of the qualifier's definition. Each variable declaration includes a type and a classifier. The declared classifier Expr for $\mathrm{E}$ indicates that during typechecking of a $\mathrm{C}$ program, $\mathrm{E}$ will be instantiated with side-effect-free program expressions.
The declared type for E constrains such expressions to have type int. In addition to the classifier Expr, our framework supports the classifiers Const, LValue, and Var, which represent $\mathrm{C}$ constants, l-values, and variables, respectively. Our implementation performs qualifier checking over programs in CIL's intermediate language, which cleanly distinguishes expressions, which are side-effect-free, from instructions [32].

Given the declaration in line 1 of figure 1, programmers may now annotate their programs with the pos qualifier, as shown in the $\mathrm{C}$ code in figure 2 . The $1 \mathrm{~cm}$ procedure in the figure computes the least-common multiple of two integers. The pos qualifier is used to specify that the two arguments should be positive integers and to ensure that the return value is also positive. To handle nested qualifiers unambiguously, we use a postfix notation, whereby a qualifier qualifies the entire type to its left. A type may be annotated with multiple user-defined qualifiers; their order is irrelevant.

\subsubsection{Type Rules}

Line 1 of figure 1 declares the new pos qualifier, but it does not indicate how this qualifier should be used during typechecking. This is the role of the case block beginning on line 2, which uses a form of pattern matching to indicate a subset of expressions that can be given the type int pos. For example, the clause in lines 34 indicates that a positive integer constant may be given the type int pos. The clause first declares the variable $C$, which ranges over integer constants from the underlying program, for use in the rest of the clause. It then specifies the pattern $C$, to indicate the syntactic form of the expression. Finally, the predicate $C>0$ further constrains an expression that matches the pattern.

Type rules like the first case clause of figure 1 can be simulated in systems like CQUAL [19] by annotating all positive integers in a program with a pos assumption. However, the case clauses in our framework are more general. For example, the clause on lines 5-6 specifies that an expression that is a product of two expressions of type int pos can also be given the type int pos. This kind of recursive type rule would be quite difficult to manually encode using pos assumptions. The final case clause illustrates that the definition of a qualifier can depend on other qualifiers. That clause specifies that a negation expression can be given type int pos if the negated expression can be given type int neg, where neg is another user-defined qualifier. In fact, qualifier definitions can be mutually recursive. For example, the definition of neg (not shown) has rules that refer to pos.

The syntax for expression patterns in case clauses is defined by the following grammar:

$$
P \quad::=\quad X|* X| \& X \mid \text { new } \mid \text { uop } X \mid X \text { bop } X
$$

Here $X$ ranges over variable patterns, which have a declared type and classifier (e.g., int Expr) restricting the kinds of program fragments that may match. The pattern new matches against calls to memory allocation routines like malloc ${ }^{1}$. Various unary and binary operations may also be matched against; for simplicity we restrict their argument patterns to be variable patterns. The predicate after (the optional) where in a case clause may include operations on constants and on variable patterns with classifier Const, qualifier checks on expressions and patterns, and conjunctions and disjunctions of these kinds of predicates.

Each clause of a case block can be viewed as an introduction type rule for a qualified type, since the clause specifies conditions under which an expression may be assigned that qualified type. As

\footnotetext{
${ }^{1}$ Procedure calls, including malloc, are not in general side-effect-free and so are not considered expressions by CIL. However, it is critical for some qualifi ers that pertain to pointers, for example unique, that we allow malloc to be matched against in qualifi er defi nitions.
} 


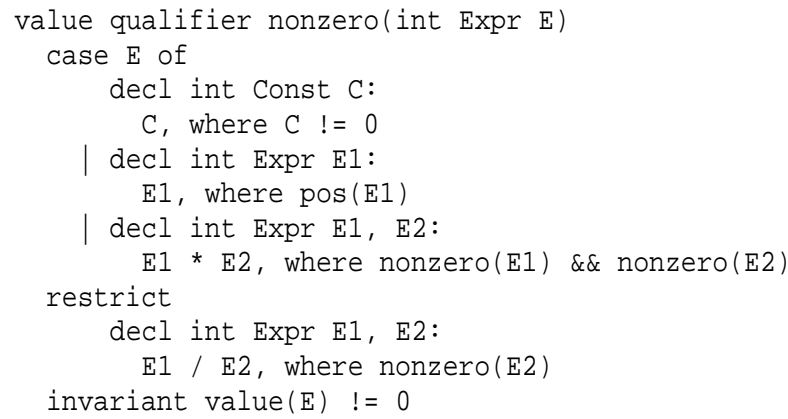

Figure 3. A type qualifier and associated type rules for nonzero integers.

discussed in section 5, we have formalized this semantics of the case clauses.

Our framework includes an extensible typechecker, which uses the type rules defined by case blocks, along with a set of standard rules for typechecking constructs like variable references, procedure calls, and assignments, to perform qualifier checking. Such checking validates the qualifier annotations supplied by the programmer, which represent the programmer's assumptions about when particular invariants hold.

Consider again the $1 \mathrm{~cm}$ procedure in figure 2. As usual, typechecking an assignment statement involves obtaining the types of each side and checking that they match. The assignment to d typechecks successfully because both sides of the assignment have type int pos: the right-hand side is shown to have this type by the standard type rule for procedure calls, given the declared type signature of gcd. The assignment to prod also typechecks successfully, because the case clause on lines 5-6 allows $a * b$ to be given the type int pos. Because of their declared types, we statically know that both prod and $\mathrm{d}$ are positive, but this information is not sufficient to show that the expression (prod / d) is also positive. Indeed, the type rules for pos are not able to derive the type int pos for that expression. Therefore, the programmer must insert a cast to satisfy the typechecker, because of the declared return type of $1 \mathrm{~cm}$.

A case block specifies when an expression may be given a qualified type. Users may then want to employ expressions having qualified types to enhance the precision of existing typechecks from the base type system. Our framework provides a restrict block for this purpose, an example of which is shown in the definition for a nonzero qualifier in figure 3 . The syntax of a restrict clause is identical to that of a case clause. A restrict clause specifies that any expression in a given program that matches the clause's pattern must also satisfy the clause's predicate. The restrict clause for nonzero augments the base rule for typechecking division expressions to require that the denominator have the type int nonzero (rather than simply int). In this way, division-by-zero errors can be detected statically instead of dynamically.

For example, consider again the $1 \mathrm{~cm}$ procedure in figure 2 . If the extensible typechecker is given the definition of nonzero in addition to that of pos, it will use nonzero's restrict clause to check the division in the last statement of $1 \mathrm{~cm}$ 's body. The restrict clause requires that $d$ have the type int nonzero. By the second case clause for nonzero in figure 3, any expression of type int pos also has the type int nonzero. Since $d$ is declared to have the type int pos, that case clause allows the restrict check to succeed.

The restrict clause plays a role analogous to qualifier assertions in CQUAL [19]. For example, the restrict type rule in figure 3 could be simulated by annotating the denominator in each division in a program with a nonzero assertion. However, the restrict clause is more general. For example, the predicate in a restrict clause may contain conjunctions and disjunctions of qualifier checks.

\subsubsection{Subtyping}

It is natural to consider int pos to be a subtype of int. Subtyping provides more flexibility, for example allowing the following code to typecheck:

int pos $x=3$;

int $\mathrm{y}=\mathrm{x}$;

Our extensible typechecker considers all value-qualified types to be subtypes of their associated unqualified types. More precisely, if $q$ is a value qualifier and $\tau$ is a (possibly qualified) type, then $\tau q$ is considered to be a subtype of $\tau$.

The rest of the subtyping rules are standard. As usual, care must be taken in the presence of pointers [19]. For example, it would be unsound to consider int pos* to be a subtype of int*, because that would allow the following code, which stores a negative number in a variable of type int pos, to typecheck:

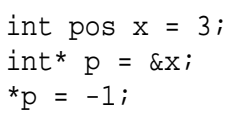

Section 5 contains the formal definition of our subtype relation.

Our language for defining qualifiers does not support explicit subtype declarations between two user-defined qualifiers. However, such subtype relationships can be encoded using the case block. For example, the second clause in the case block of nonzero's definition in figure 3 effectively declares pos to be a subtype of nonzero: any expression of type int pos may also be considered to have type int nonzero.

\subsubsection{Soundness}

A user-defined qualifier and its associated type rules constitute a typing discipline, which is enforced by our extensible typechecker. Often such typing disciplines are intended to ensure a particular run-time invariant. For example, the typing discipline defined by the pos qualifier and associated type rules in figure 1 is intended to guarantee that certain expressions only evaluate to positive integers at run time.

However, the extensible typechecker enforces user-defined typing disciplines in a purely syntactic manner, without knowledge of the intended invariants. For example, suppose the pattern in the second case clause in the definition of pos in figure 1 were erroneously specified as E1 - E2 instead of E1 * E2. In that case, our typechecker would happily use this revised type rule to check programs, even though this can cause pos's intended invariant to be violated at run time.

Rather than forcing users to take responsibility for the correctness of their qualifiers, our framework supports automated soundness checking. A qualifier definition may optionally specify the qualifier's associated invariant. The framework then automatically proves, independent of any particular program to be typechecked, that the qualifier's type rules establish this invariant.

For example, consider the definition of pos in figure 1. Line 9 uses the invariant clause to provide the qualifier's associated run-time invariant. The invariant is a predicate that is implicitly defined in the context of an arbitrary run-time execution state. Let us denote this execution state by $\rho$. The value predicate is provided by our framework and represents the value of a given expression in $\rho$. Therefore, the invariant for pos indicates that the value of an expression of type int pos should be greater than zero, in any run-time execution state. 


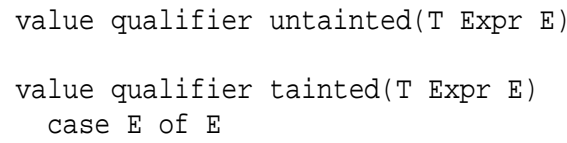

Figure 4. Specifying a taintedness analysis in our framework.

Given this invariant, our soundness checker generates one proof obligation for each case clause and automatically discharges these obligations via an off-the-shelf automatic theorem prover. Each clause's obligation simply requires that if an expression matches the clause's syntactic pattern and satisfies the clause's predicate, interpreted in the context of an arbitrary run-time execution state $\rho$, then the qualifier's invariant also holds in $\rho$. For example, consider the first case clause for pos in figure 1. The soundness checker generates the following proof obligation: if an expression $\mathrm{E}$ is an integer constant that is greater than zero, then the value of $E$ in an arbitrary execution state $\rho$ is greater than zero. This obligation is easily proven, given the evaluation semantics of integer constants.

Now consider the second case clause for pos. The soundness checker generates the following proof obligation: if an expression $E$ has the form E1 * E2 and both E1 and E2 satisfy pos's invariant in an arbitrary execution state $\rho$, then $\mathrm{E}$ also satisfies pos's invariant in $\rho$. This obligation is easily proven by the semantics of multiplication. On the other hand, if the pattern in that clause were erroneously specified as E1 - E2, the soundness checker would catch the error and warn the programmer, since the associated proof obligation would fail: it is not possible to prove that the difference of two arbitrary positive integers is also positive. The restrict clauses do not affect whether or not an expression of qualified type satisfies its qualifier's invariant, so restrict clauses are ignored by the soundness checker.

The limitations of our language for writing type rules, and of static typechecking in general, will sometimes require programmers to insert casts in order for qualifier checking to succeed. To retain soundness in this case, our extensible typechecker instruments programs with a run-time check for each cast to a value-qualified type. Each run-time check tests whether the expression being cast satisfies the cast-to qualifier's invariant. In our current implementation, a fatal error is signaled if the test fails. For example, consider the cast in the last statement of $1 \mathrm{~cm}$ in figure 2 . At run time, a check ensures that the value of (prod / d) is in fact greater than zero.

\subsubsection{Flow Qualifiers}

Some common kinds of qualifiers are used solely to restrict the flow of values in a program. For example, a taintedness analysis uses qualifiers untainted and tainted to respectively tag data coming from trustworthy and potentially untrustworthy sources. For soundness, the only requirement is that tainted data never flows where untainted data is expected. Taintedness qualifiers can help to statically detect format-string vulnerabilities in calls to printf and related procedures [36]. As another example, flow qualifiers user and kernel can be used to statically ensure that user pointers are never dereferenced in kernel space [24].

Figure 4 specifies the taintedness analysis in our framework. The untainted qualifier can qualify any type T. Flow qualifiers like untainted are a degenerate form of value qualifier in our framework. Since the untainted qualifier has no case block, the only way to introduce an expression of type $T$ untainted is with a cast, to explicitly mark the expression as being trustworthy. The qualifier also lacks an explicit run-time invariant. Proper value flow is guaranteed "for free" because $\mathrm{T}$ untainted is a subtype of $\mathrm{T}$ but not vice versa. Therefore, untainted data can flow where arbitrary data is expected, but not vice versa.

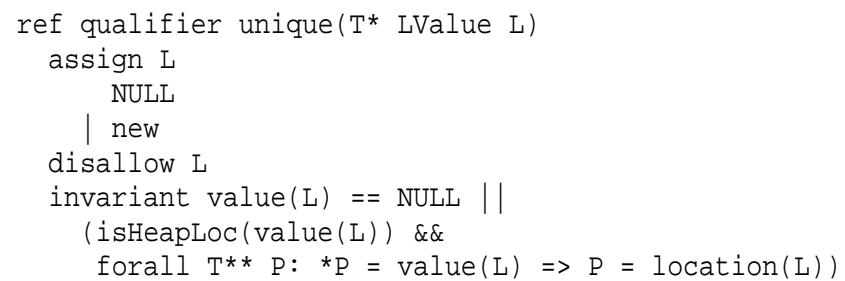

Figure 5. A type qualifier for unique pointers.

As mentioned earlier, the untainted qualifier can help find errors in calls to print $f$ and related procedures. The first argument to printf is a format string that specifies the number and types of the remaining arguments. $\mathrm{C}$ does not verify, either at compile time or run time, that print $f$ is always called with the appropriate number and types of arguments, as directed by the format-string argument. To ensure some measure of reliability for format strings, the programmer can use a type signature for printf requiring the first formal parameter to have type char* untainted. Therefore, only strings that are known to be trustworthy can be used as formatstring arguments [36].

For example, suppose buf is some arbitrary (and untrusted) buffer of type char*. Then the following code typechecks:

char* untainted fmt $=$ (char* untainted) "\%s"; printf (fmt, buf);

However, the invocation printf (buf) fails to typecheck, since buf is not known to be untainted. Indeed, if buf contains format specifiers, this call to printf will attempt to read nonexistent arguments off the stack.

It may be useful to explicitly annotate some expressions as being possibly tainted. The definition of tainted in figure 4 has the desired behavior. The lone case clause allows any expression to be considered tainted, effectively making $\mathrm{T}$ tainted a supertype of $\mathrm{T}$ (and hence also of $\mathrm{T}$ untainted). Because of the implicit subtyping relation for value qualifiers, it is also the case that $T$ tainted is a subtype of $\mathrm{T}$, so those types are essentially equivalent.

Although the versions of tainted and untainted in figure 4 are degenerate, they could easily be augmented. For example, a user could decide that all constants should be trusted, adding a case clause to the definition of untainted as follows:

case $\mathrm{E}$ of decl $\mathrm{T}$ Const $\mathrm{C}$ : C

This rule would, for example, obviate the need for the cast in the assignment to fmt in the code snippet shown above.

\subsection{Reference Qualifiers}

Figure 5 defines a reference qualifier unique, which intuitively specifies that an l-value either has the value NULL or is the only reference to some memory location. Reference qualifiers pertain in part to an expression's address, so we require each reference qualifier to be applicable only to l-values or variables, rather than to arbitrary expressions.

\subsubsection{Type Rules}

The assign type rules for reference qualifiers are analogous to the case type rules for value qualifiers. The assign block allows users to specify the allowable right-hand-side expressions in assignments to a qualified l-value. These type rules are also used to typecheck implicit assignments to qualified l-values, through procedure calls and returns.

The assign clauses for unique in figure 5 specify that a unique l-value may be assigned either the value NULL or the result of mem- 


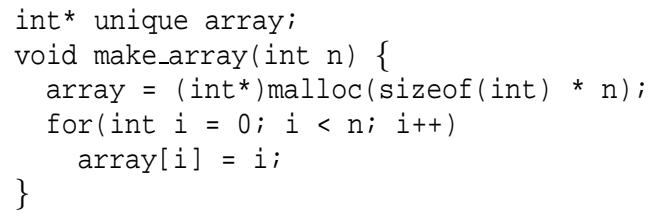

Figure 6. Code that uses the unique qualifier.

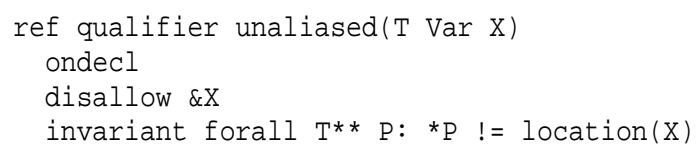

Figure 7. A type qualifier for unaliased variables.

ory allocation. Consider the code in figure 6 . The assignment to array in make_array typechecks by the second assign clause, since C's malloc function matches the pattern new (the cast to int* in the assignment to array is ignored for the purposes of pattern matching). The assign block for unique pertains only to assignments to the unique 1-value itself. Assignments to dereferences of a unique 1-value, such as the assignment to array [i] in figure 6, are unrestricted.

Because reference qualifiers pertain in part to an 1-value's address, they do not make sense in the context solely of an 1-value's contents. Therefore, top-level reference qualifiers in an l-value's type are not considered to be part of the l-value's r-type, which is the type used by the typechecker when the l-value appears on the right-hand side of an assignment (and in other expressions like conditionals). However, it may still be necessary to restrict the ways in which an l-value's contents may be used, to ensure soundness. For example, without any restrictions, the following code snippet would typecheck:

int* unique $\mathrm{p}=\ldots$;

int* $\mathrm{q}=\mathrm{p}$;

The second statement is allowed because the r-type of $p$ is simply int*, but it causes $p$ and $q$ to point to the same memory location, violating p's uniqueness.

The disallow clause in a reference qualifier's definition addresses this problem by restricting how a qualified 1-value may be used on the right-hand side of an assignment (or an implicit assignment via procedure calls and returns). A disallow clause may prevent a qualified l-value from being referred to and/or from having its address taken. The disallow clause for unique in figure 5 prevents a qualified 1-value from being referred to. Therefore, the assignment to $q$ in our code snippet above fails to typecheck, as desired, since the right-hand side violates this disallow clause. A unique l-value may still be dereferenced on the right-hand side of an assignment, so the following code typechecks and is perfectly safe:

int* unique $\mathrm{p}=\ldots$;

int $i={ }^{*} p$;

Another example of a reference qualifier is shown in figure 7, representing unaliased variables. The ondecl keyword specifies that any variable can be given the qualifier unaliased at the point of its declaration. The keyword indicates that unaliased is a property only of a variable's address; the variable's contents are irrelevant. Therefore, an assign block is unnecessary for unaliased: implicitly, an unaliased variable is allowed to hold any type- correct value. The disallow clause for unaliased ensures that an unaliased variable cannot have its address taken.

Our current framework for reference qualifiers is just a first step, and we are actively exploring extensions to make it more expressive. For example, intuitively we can assign a unique 1value any expression that is fresh, meaning that the expression evaluates to a previously unreferenced memory location. In turn, a unique local variable returned from a procedure may be considered fresh. We cannot currently express this rule in our framework because patterns cannot mention procedure calls or returns. As another example, we are considering allowing qualifier definitions to directly refer to liveness information. This would allow, for example, a variable to safely be considered unique as long as all aliases are dead [5].

\subsubsection{Subtyping}

Unlike for value qualifiers, there is in general no sound sub- or supertype relationship between an arbitrary reference-qualified type and its associated unqualified type. Therefore, we assume no relationship between these two types. However, the implicit stripping of reference qualifiers from the r-type of an 1-value, as described earlier, allows expressions of these types to interact in useful (and still sound) ways.

\subsubsection{Soundness}

As with value qualifiers, our framework automatically proves the soundness of reference qualifiers. Consider the invariant clause for unique in figure 5. The built-in location function returns the address of an l-value in a given execution state $\rho$, and the builtin predicate isHeapLoc indicates that a memory location is on the heap (i.e., it was dynamically allocated) rather than the stack. We allow the invariants for reference qualifiers to universally quantify over all memory locations $P$ of the appropriate type in the execution state $\rho$, and ${ }^{*} \mathrm{P}$ denotes the contents of location $\mathrm{P}$ in $\rho$.

The assign type rules are proven sound in a manner analogous with how the case rules are proven sound for value qualifiers. The proof obligation for an assign clause ensures that assigning some l-value $l$ an expression matching the assign clause establishes $q$ 's associated invariant for $l$. For example, consider the first assign clause for unique in figure 5 . The soundness checker requires an automatic theorem prover to show that if some l-value $l$ is assigned NULL, then unique's invariant will hold for $l$. This follows easily by the first disjunct in the invariant. The obligation for the second assign clause is proven by the semantics of the new construct. The proof obligation for ondecl is similar to that for an assign clause, requiring the qualifier's invariant to hold for a variable upon declaration.

The proof obligations for assign and ondecl ensure that a reference qualifier's invariant is properly established. Our soundness checker must also show that the invariant is properly preserved across assignments. Accordingly, we generate the following proof obligation: if $l$ is an l-value satisfying the invariant of some reference qualifier $q$ and we execute an arbitrary assignment to some other l-value $l^{\prime}$, then $l$ will still satisfy the qualifier's invariant in the resulting execution state. We require the arbitrary assignment considered in the obligation to obey the disallow block for $q$, if any is specified.

The automatic theorem prover proves this obligation via a case analysis on the different forms of right-hand sides consistent with a qualifier's disallow clause. If the disallow clause for unique were erroneously omitted, one case in proving unique's preservation obligation would require showing that if $l$ is unique and we store the value of $l$ in $l^{\prime}$, then $l$ is still unique. Because this case is not provable, the soundness checker would correctly inform the user of the potential unsoundness. 
Unlike for value qualifiers, our extensible typechecker does not instrument programs with run-time checks for casts involving reference qualifiers. Such casts remain unchecked, as with traditional casts in C. As our examples illustrate, the invariants for reference qualifiers typically require universal quantification over all memory locations, making the associated run-time checks difficult to implement correctly and efficiently.

\section{Extensible Typechecking}

Our extensible typechecker takes a $\mathrm{C}$ program and a set of qualifier definitions in the language described in the previous section. The extensible typechecker then performs qualifier checking on the program as directed by the qualifier definitions' type rules. The extensible typechecker also uses value qualifiers' declared invariants to instrument the program with run-time checks for casts involving value qualifiers, as mentioned in section 2.1.3.

Our extensible typechecker is implemented as a module in CIL [32], a front end for C written in OCaml [35]. CIL parses $\mathrm{C}$ code into an abstract syntax tree (AST) format and provides a framework for performing passes over this AST. After qualifier checking, the AST is output as C code and the gcc compiler performs ordinary $\mathrm{C}$ typechecking and code generation.

In this section, we discuss the implementation of our extensible typechecker. First we describe how $\mathrm{C}$ programs are annotated with user-defined qualifiers. Then we illustrate how user-defined type rules are represented and used in our CIL implementation. Finally, we discuss some details of handling $\mathrm{C}$ programs.

\subsection{Annotating Programs}

To annotate a $\mathrm{C}$ program with qualifiers, we take advantage of gcc attributes, which are tags that can be associated with types (and other program entities). CIL supports gcc attributes and maintains them in the generated AST for a program. A type attribute follows the type name and has the following syntax:

--attribute_- ( (attribute name) )

We typically use macros instead of writing the unwieldy attribute syntax directly. Such macros are used in our examples of section 2 . For example, the qualifier pos used in figure 2 is defined as follows:

\#define pos _-attribute_-( (pos))

\subsection{Qualifier Checking with CIL}

Our qualifier checker traverses the given CIL AST, applying userdefined type rules to applicable program fragments. Any type errors found during qualifier checking are provided to the programmer as warnings, but compilation is allowed to continue.

To implement qualifier checking, we have created OCaml datatypes to represent the expression patterns and predicates that are allowed in user-defined type rules. For example, consider the case clause on lines 5-6 in figure 1 . The expression pattern is represented internally as follows:

Binop (Mult, Expr("E1"), Expr("E2"))

The clause's predicate is similarly represented as follows:

And (Qual ("pos", Expr ("E1")),

Qual ("pos", Expr("E2")))

Consider the application of this type rule to the right-hand side of the assignment to prod in figure 2. First we match our expression pattern against the CIL AST for $a * b$. The match succeeds and produces bindings for variables in the pattern: $\mathrm{E} 1$ is bound to the expression a and E2 is bound to the expression b. Finally, the rule's predicate is evaluated, after replacing each pattern variable with the $\mathrm{C}$ program fragment to which it is bound. In our example, the predicate is satisfied if $a$ and $b$ can recursively be given the qualifier pos. The other kinds of type rules are represented and checked similarly.

\subsection{Interacting with $\mathbf{C}$}

We allow types to be annotated with qualifiers wherever they appear. For example, the types of struct fields may be qualified, and our qualifier checker will check that they obey the user-defined type rules. Fields of unions may also be given qualified types, but the usual unsoundness for $\mathrm{C}$ unions makes our qualifier checking in this case unsound as well.

As is often the case for $\mathrm{C}$ program analyses, we assume a logical model of memory. In particular, we assume that the type of $p+i$, where $p$ is a pointer and $i$ is an integer, is the same as the type of p. This assumption is unsound, but in practice it removes a large source of spurious type errors, for example arising from pointer arithmetic for array indexing.

Another source of spurious type errors arises from invoking procedures in the $\mathrm{C}$ standard library, since their argument and result types are not annotated with user-defined qualifiers. We currently solve this problem by writing header files that contain alternate signatures for library procedures, which replace the procedures' ordinary signatures via gcc command-line macros. We plan to develop a standardized mechanism for incorporating qualifier annotations in library code as directed by user-provided specification files.

Macros from the standard library are also problematic. When these macros are expanded by the $\mathrm{C}$ preprocessor, our qualifier checker produces type errors because the macros' bodies are not properly annotated. Short of creating our own versions of these macros, we have little recourse.

Our qualifier checker can also be unsound because it, like C, allows variables to be used before being initialized. Finally, our checker is unsound in the presence of arithmetic overflow.

Because of these unsoundnesses, our extensible typechecker for $\mathrm{C}$ can be used to statically detect potential errors but cannot guarantee the absence of errors of a particular kind. However, the ideas underlying our framework are not specific to $C$ and could be applied to other languages. For example, a version of our extensible typechecker for a typesafe language like Java [2, 22] would remove most of these sources of unsoundness.

\section{Automated Soundness Checking}

Our soundness checker takes a qualifier definition, generates the necessary proof obligations for each user-defined type rule, and automatically discharges these obligations using Simplify [14], a Nelson-Oppen-style automatic theorem prover [34]. Simplify contains decision procedures for several decidable theories, including linear arithmetic and equality for uninterpreted function symbols. Simplify's input language accepts first-order formulas over these theories.

This section details the implementation of our soundness checker. First we describe the axioms we provide Simplify so it can reason about $\mathrm{C}$ programs, and then we describe the obligations that are proven in the context of these axioms. We have used our soundness checker to automatically prove the soundness of a variety of type qualifiers. The value qualifiers nonnull, nonzero, pos, and neg are each proven sound by our checker in under one second. The reference qualifiers unique and unaliased are each proven sound in under 30 seconds.

\subsection{Axioms}

We use axioms to formalize the dynamic semantics of programs in CIL's intermediate language. The state of a program is represented by an execution state $\rho=(\pi, 1, \varepsilon, \sigma)$, where $\pi$ is a program, $\imath$ is 
an index pointing to the statement about to be executed, $\varepsilon$ is the environment, which maps variable names to memory locations, and $\sigma$ is the store, which maps locations to values.

We define several function symbols for constructing and manipulating execution states. The state function symbol takes a program, index, environment, and store, and it constructs an execution state. The function symbols getStmt, getEnv, and getStore take a state and respectively return the statement about to be executed, the environment, and the store. Environments and stores are represented as maps. Simplify has built-in function symbols that represent operations on maps. For example, the built-in select function symbol takes a map and a key and returns the key's associated value. Finally, we represent $\mathrm{C}$ program expressions and statements using additional function symbols. For instance, the statement $* x:=\& y$ is encoded as $\operatorname{assign}(\operatorname{deref}(\operatorname{var}(x)), \operatorname{addr}(\operatorname{var}(y)))$.

Given this representation, we define axioms for a function symbol evalExpr, which evaluates an expression in a given state. For instance, the following axiom formalizes evaluation of variable references $^{2}$ :

$$
\begin{aligned}
& \forall \rho, e, x .(e=\operatorname{var}(x) \Rightarrow \\
& \quad \operatorname{evalExpr}(\rho, e)=\operatorname{select}(\operatorname{getStore}(\rho), \operatorname{select}(\operatorname{get} \operatorname{Env}(\rho), x)))
\end{aligned}
$$

We similarly define axioms for a function location, which takes an l-value and returns its address, and a function stepState, which takes a program state and returns the state resulting from executing the current statement.

Our axioms only formalize the subset of the CIL intermediate language necessary for reasoning about expression patterns. For example, we do not currently axiomatize the semantics of procedure calls, since they cannot be pattern-matched against. We do, however, explicitly model memory allocation, via a new function symbol.

\subsection{Proof Obligations}

To produce a qualifier's proof obligations, first we define a predicate to represent the qualifier's invariant. Built-in function symbols like value in qualifier definitions are translated to their counterpart function symbols in the axioms. For example, the invariant for pos from figure 1 is defined as follows:

$$
\operatorname{pos}(\rho, e)=(\operatorname{evalExpr}(\rho, e)>0)
$$

Proving the soundness of a qualifier $q$ also requires access to the invariants of all qualifiers $q^{\prime}$ that are referred to in $q$ 's type rules.

Given these invariants it is straightforward to represent our proof obligations in Simplify. For example, the obligation for the second case clause of pos in figure 1 is defined as follows:

$\forall \rho, e_{1}, e_{2} \cdot(\operatorname{pos}(\rho, e 1) \wedge \operatorname{pos}(\rho, e 2)) \Rightarrow \operatorname{pos}(\rho, \operatorname{multExpr}(e 1, e 2))$

As another example, the obligation for the second assign clause of unique in figure 5 is defined as follows:

$\forall \rho, l .(\operatorname{getStmt}(\rho)=\operatorname{assign}(l$, new $)) \Rightarrow$ unique $(\operatorname{stepState}(\rho), l)$

\section{Formalization}

We have formalized our extensible type system for value qualifiers and have proven that the obligations generated by our soundness checker are sufficient to ensure type soundness for such qualifiers. This section overviews these formal details; the complete formalization for value qualifiers is available in a companion technical report [7].

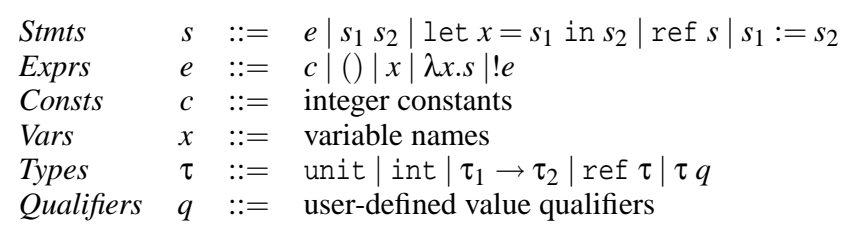

Figure 8. The syntax of the formalized language.

$$
\begin{gathered}
\overline{\tau q \leq \tau} \text { SubValQual } \frac{}{\tau q_{1} q_{2} \leq \tau q_{2} q_{1}} \text { SubQualReorder } \\
\overline{\tau \leq \tau} \text { SUbReF } \frac{\tau \leq \tau^{\prime \prime} \quad \tau^{\prime \prime} \leq \tau^{\prime}}{\tau \leq \tau^{\prime}} \text { SuBTRans } \\
\frac{\tau_{1}^{\prime} \leq \tau_{1} \quad \tau_{2} \leq \tau_{2}^{\prime}}{\tau_{1} \rightarrow \tau_{2} \leq \tau_{1}^{\prime} \rightarrow \tau_{2}^{\prime}} \text { SUBFUN }
\end{gathered}
$$

Figure 9. Formal rules for subtyping.

$$
\begin{gathered}
\Gamma \vdash e: \tau \\
\frac{\Gamma \vdash e_{1}: \tau_{1} q_{1} \quad \ldots \quad \Gamma \vdash e_{n}: \tau_{n} q_{n}}{\text { each } e_{i} \text { is a subexpression of } e} \\
\Gamma \vdash e: \tau q
\end{gathered}
$$

Figure 10. A formal template for user-defined case clauses.

\subsection{Syntax and Semantics}

We formalize our framework in the context of a simply-typed lambda calculus augmented with ML-style references [29] and user-defined type qualifiers. The syntax for this language is shown in figure 8. It is useful to distinguish statements, which are potentially side-effecting, from expressions, which are side-effect-free, but this separation causes no loss of expressiveness.

The static semantics is defined, as usual, by a judgment of the form $\Gamma \vdash s: \tau$, where $\Gamma$ is a type environment mapping variable names to types. The inference rules defining this judgment include all the standard rules for the simply-typed lambda calculus with references. We augment these rules with the standard subsumption rule and with an associated subtyping relation, which is shown in figure 9. Rule SUB VALQUAL allows a value-qualified type to be a subtype of the associated unqualified type. Rule SUBQUALREORDER formalizes the fact that the order of qualifiers on a type is irrelevant. The other rules are standard. There is no rule for subtyping underneath ref types, so the type ref $\tau$ is only a subtype of itself.

To complete the static semantics, figure 10 formalizes the userdefined case clauses in a value qualifier's definition. The rule TQUALCASE is actually a rule template: each qualifier is assumed to have zero or more rules of this form. The template allows an expression to be given a qualified type if the expression has the associated unqualified type and some of the expression's subexpressions have particular qualified types. For example, the second case clause in the definition of pos in figure 1 would be formalized by the following rule, which matches the template:

\footnotetext{
${ }^{2}$ Throughout this section, we elide portions of axioms and proof obligations that involve "typing predicates," which are used to restrict the domains of function symbols.
} 


$$
\begin{aligned}
& \overline{\Gamma \text {;int } \vdash<\sigma, c\rangle} \text { Q-InT } \overline{\Gamma \text {;unit } \vdash<\sigma,()>} \text { Q-UNIT } \\
& \frac{\Gamma \vdash \lambda x . s: \tau_{1} \rightarrow \tau_{2}}{\Gamma ; \tau_{1} \rightarrow \tau_{2} \vdash<\sigma, \lambda x . s>} \text { Q-FUN } \\
& \frac{\Gamma \vdash l: \operatorname{ref} \tau \quad \Gamma ; \tau \vdash<\sigma, \sigma(l)>\quad l \in \operatorname{dom}(\sigma)}{\Gamma ; \operatorname{ref} \tau \vdash<\sigma, l>} \text { Q-REF } \\
& \frac{[[q]](v) \quad \Gamma ; \tau \vdash<\sigma, v>}{\Gamma ; \tau q \vdash<\sigma, v>} \text { Q-QUAL }
\end{aligned}
$$

Figure 11. Semantic conformance of a value to a type.

$$
\begin{gathered}
\Gamma \vdash e_{1} * e_{2}: \text { int } \bar{q} \\
\frac{\Gamma \vdash e_{1}: \text { int pos } \Gamma \vdash e_{2}: \text { int pos }}{\Gamma \vdash e_{1} * e_{2}: \text { int } \bar{q} \text { pos }}
\end{gathered}
$$

The restrict rules of a value qualifier do not affect soundness, so we have not included these rules in our formalization.

The dynamic semantics of our language is completely standard and is formalized via a big-step operational semantics. Programs are evaluated to values defined by the following grammar:

$$
\begin{array}{llll}
\text { Values } & v & := & c|()| \lambda x . s \mid l \\
\text { Locations } & l & := & \text { memory locations }
\end{array}
$$

A store $\sigma$ maps memory locations to values. The evaluation relation has the form $\langle\sigma, s\rangle \rightarrow\left\langle\sigma^{\prime}, v\right\rangle$, indicating that evaluating statement $s$ in store $\sigma$ produces value $v$ and a new store $\sigma^{\prime}$. Since expressions are side-effect-free, their evaluation is formalized by a relation of the form $\langle\sigma, e\rangle \rightarrow v$.

\subsection{Invariants and Proof Obligations}

We model a value qualifier $q$ 's associated invariant as a unary predicate on values, denoted $[[q]]$. For example, the invariant clause for pos in figure 1 would be formalized as the predicate $[[$ pos $]](v) \equiv v>0$. We overload the $[[q]]$ notation to lift these predicates from values to arbitrary expressions:

$$
[[q]](\sigma, e, v) \equiv(<\sigma, e>\rightarrow v \Rightarrow[[q]](v))
$$

Finally, we formalize the proof obligation generated by our soundness checker for each user-defined type rule:

DEFINITION 5.1. A type rule matching the template $\mathrm{T}$ QUALCASE from figure 10 is locally sound if the following proof obligation is true:

$$
\begin{aligned}
& \forall \sigma, v_{1}, \ldots, v_{n}, v . \\
& \quad\left(\left[\left[q_{1}\right]\right]\left(\sigma, e_{1}, v_{1}\right) \wedge \ldots \wedge\left[\left[q_{n}\right]\right]\left(\sigma, e_{n}, v_{n}\right)\right) \Rightarrow[[q]](\sigma, e, v)
\end{aligned}
$$

\subsection{Type Soundness}

We have proven the soundness of our extensible type system for value qualifiers. Intuitively, soundness means that if the proof obligations generated by our soundness checker are all true, then any well-typed program fragment will satisfy its qualifiers' invariants at run time.

We formalize this notion of type soundness via a few auxiliary definitions. The relation $\Gamma ; \tau \vdash\langle\sigma, v\rangle$, defined in figure 11, represents semantic conformance of a value to a type. Intuitively, $\Gamma ; \tau \vdash\langle\sigma, v\rangle$ holds if $\Gamma \vdash v: \tau$ and $v$ additionally satisfies all of the associated invariants for qualifiers in $\tau$. The first three rules in figure 11 are the standard typechecking rules for integers, unit, and functions, respectively. Rule Q-QUAL checks that a value of qualified type satisfies the qualifier's invariant. Rule Q-REF checks that

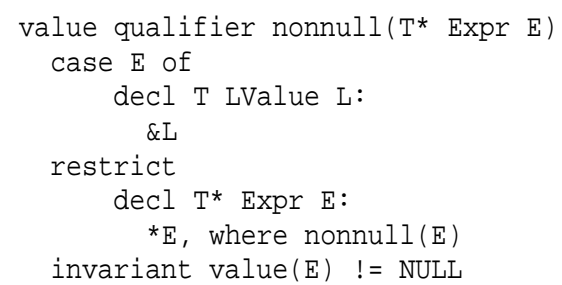

Figure 12. The nonnull value qualifier.

a location $l$ is well-typed and recursively checks semantic conformance of the value that $l$ points to in the given store. As others have done [19], for purposes of the static semantics we treat locations as variables.

Next we lift this notion of semantic conformance to a relation between a store and a type environment:

DEFINITION 5.2. We say that $\Gamma \sim \sigma$ if both of the following conditions hold:

1. $\operatorname{dom}(\Gamma)=\operatorname{dom}(\sigma)$

2. $\forall l \in \operatorname{dom}(\Gamma) .(\Gamma ; \Gamma(l) \vdash<\sigma, l>)$

In other words, $\Gamma \sim \sigma$ if every memory location is well typed and satisfies its qualifiers' invariants.

Finally we can state our type soundness theorem, which is a variant of the standard type preservation theorem [39]:

THEOREM 5.1. If $\Gamma \sim \sigma$ and $\Gamma \vdash s: \tau$ and $\langle\sigma, s\rangle \rightarrow\left\langle\sigma^{\prime}, v>\right.$ and all user-defined type rules are locally sound, then there exists some $\Gamma^{\prime} \supseteq \Gamma$ such that $\Gamma^{\prime} \sim \sigma^{\prime}$ and $\Gamma^{\prime} ; \tau \vdash<\sigma^{\prime}, v>$.

The proof of this theorem can be found in our technical report [7].

\section{Experience}

This section reports on experience using our framework for userdefined type qualifiers. We describe its usage on existing $\mathrm{C}$ programs to statically detect NULL dereferences, violations of uniqueness invariants, and improper format strings. In all of the experiments described below, the extra compile time for performing qualifier checking in CIL is under one second.

\subsection{Null Dereferences}

Figure 12 shows the definition of a nonnull value qualifier, which is automatically proven sound by our soundness checker. The sole case clause indicates that the address of an 1-value can be considered nonnull. The restrict clause requires all dereferences in a program to be to nonnull expressions.

We used this nonnull qualifier to statically ensure the absence of NULL dereferences in the grep search utility program (version 2.5). We annotated the files dfa.c and dfa.h, which comprise the core string-matching algorithm and related data structures. The files consist of 2287 non-blank, non-comment lines of code.

We applied nonnull annotations to variables in an iterative fashion. Running our extensible typechecker on the unannotated files produced an error message for each dereference, due to the nonnull qualifier's restrict clause. These errors were removed by annotating some variables with nonnull, which could in turn cause error messages on assignments to the newly-annotated variables, leading to more annotations. In addition to formal parameters and local variables, we documented several fields of structures as being nonnull through this process.

There were situations where the type rules for nonnull were insufficient and we were forced to insert casts. The major source of 
Table 1. Results from the nonnull experiment.

\begin{tabular}{|r|l|}
\hline program: & grep \\
files: & dfa.c, dfa.h \\
lines: & 2287 \\
dereferences: & 1072 \\
annotations: & 114 \\
casts: & 59 \\
errors: & 0 \\
\hline
\end{tabular}

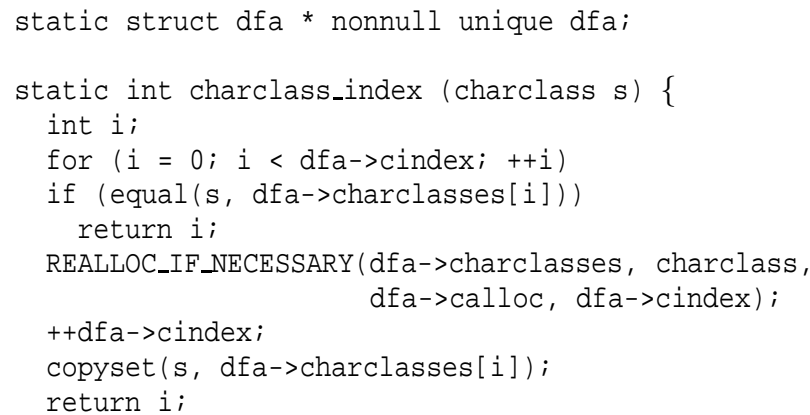

Figure 13. A use of unique in grep.

such imprecision is due to the flow-insensitivity of our type system. An example from grep follows:

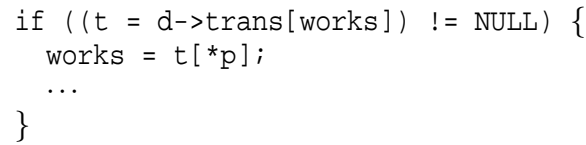

The index into array $t$ is safe because it is guarded by the check for NULL, but our type system cannot deduce this fact. We plan to extend our typechecking algorithm to incorporate flow-sensitivity, borrowing ideas from CQUAL [20].

A related source of imprecision occurs when access to a NULLterminated array is guarded by a test that the index is less than the value of a variable holding the array's length. Statically deducing the invariant between the array and that variable may be difficult. One possibility would be to piggyback our qualifier checker on top of CCured [33], which (among other things) can sometimes statically deduce array bounds.

Table 1 summarizes the results of our experiment. In order for the restrict clause in nonnull to succeed on all 1072 dereferences, we had to provide 114 nonnull annotations and 59 nonnull casts.

\subsection{Uniqueness}

The implementation of grep makes use of several global data structures, which are manipulated by a variety of procedures. It would be nice to statically ensure that each global variable is the sole reference to the data structure to which it points. In this way, we can guarantee that each data structure cannot be updated unexpectedly through other pointers.

We annotated several global variables in dfa. $c$ with the unique qualifier, using the definition of unique from figure 5 . We had the most success with the global variable dfa, which contains the current deterministic finite-state automaton (DFA) being constructed. Our assign rules were not sufficient to statically validate the initialization of $\mathrm{dfa}$, since it is initialized to a pointer passed in from the parser module. However, the extensible typechecker validated
Table 2. Results from the untainted experiment.

\begin{tabular}{|r|l|l|l|}
\hline program: & bftpd & mingetty & identd \\
lines: & 750 & 293 & 228 \\
printf calls: & 134 & 23 & 21 \\
annotations: & 2 & 1 & 0 \\
casts: & 0 & 0 & 0 \\
errors: & 1 & 0 & 0 \\
\hline
\end{tabular}

all 49 subsequent references to dfa as preserving the variable's uniqueness. Our annotated declaration of dfa and a representative procedure manipulating dfa are shown in figure 13.

Other global variables were not able to be proven unique using our qualifier, because the variables are passed as arguments to procedures. This idiom violates the disallow clause for unique in figure 5. Indeed, this idiom is a violation of uniqueness: inside a procedure where a global is passed, the global is no longer unique. It is possible that we could statically check this idiom by relaxing our unique qualifier to support "lent" references [1], which allow a unique reference to be temporarily aliased.

\subsection{Untainted Format Strings}

Our final experiment used the untainted qualifier to ensure proper format-string arguments to printf. We used the simple version of untainted defined in figure 4 , augmented with a case clause that defines all constants to be untainted:

case $\mathrm{E}$ of decl $\mathrm{T}$ Const $\mathrm{C}$ : $\mathrm{C}$

We used this qualifier to annotate and check three of the programs tested by Shankar et al. [36], who performed a taintedness analysis using CQUAL. The programs are bftpd (version 1.0.11), an FTP server; mingetty (version 0.9.4), a remote terminal utility; and identd (version 1.0), a network identification service. For all three programs, we were able to reproduce the results of Shankar et al.

Our results are shown in table 2. Running our qualifier checker on bftpd indicated two procedure parameters that are necessary to annotate as untainted, since they are used as format strings for printf. Re-running the qualifier checker then revealed an exploitable error that had been previously identified $[3,36]$. The offending code is shown below:

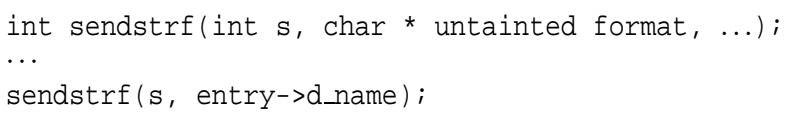

The d_name field of entry is a file name and should not be considered a proper format string. The extensible typechecker appropriately signals an error since the field has not been declared untainted.

The other two test programs were verified to have no formatstring vulnerabilities. In addition, no casts were required for any of the three test programs; the simple case clause defined above was sufficient to infer the untaintedness of all format-string arguments.

\section{Related Work}

Our framework is inspired by the CQUAL system of Foster et al. [19]. As mentioned in the introduction, our language for userdefined type rules is novel; a limited form of type rules is simulated in CQUAL via qualifier assertions and assumptions. CQUAL also does not support automated soundness checking to ensure that a qualifier establishes its intended invariant. Like our system, CQUAL distinguishes between value and reference qualifiers. CQUAL supports explicit subtyping relationships among qualifiers, which our framework lacks. However, subtyping in CQUAL is declared by 
the programmer and trusted to be correct, while we prove the semantic soundness of subtyping in our framework. CQUAL supports qualifier inference and qualifier polymorphism, neither of which our framework supports. Follow-on work extended CQUAL's type system to be flow-sensitive [20], while our type system is flowinsensitive.

The type-refinement framework of Mandelbaum et al. [27] supports a sophisticated type system that is flow sensitive and precise in the face of computational effects, allowing temporal protocols similar to those expressible in the Vault language [12] to be statically checked. Both the framework of Mandelbaum et al. and Vault also support a form of polymorphism for refinements. However, those systems lack a language for user-defined type rules and lack automated soundness checking with respect to run-time invariants.

Fugue [13] is an adaptation and extension of Vault's type system to perform static checking of temporal protocols for C\# [6]. Among other innovations, Fugue allows a class's typestates, which are the analogues of our qualifiers, to be given an interpretation as a predicate over the class's fields. Such predicates are used during static typechecking to ensure that each method in the class properly implements its declared specification. In this way, the Fugue typechecker directly ensures that typestates respect their intended invariants. These invariants appear to be similar in expressiveness to ours, except that Fugue's predicate language does not support quantification. Further, Fugue's type system relies heavily on builtin annotations and associated type rules for nonnull references as well as for several kinds of aliasing relationships among objects, while these kinds of qualifiers are user-defined in our framework.

Some type systems, including the calculus of constructions [10], Nuprl [9], and type systems [37, 11] for Proof-Carrying Code (PCC) [31] and Typed Assembly Language [30], use a form of dependent types [28] to allow predicates to be directly encoded as types. However, the proof that a predicate holds on some program fragment cannot in general be produced automatically and must instead be supplied by the programmer. Our framework is less expressive than these systems, since predicates can only be proven indirectly via type qualifiers and their associated type rules. However, the separation of typechecking, which is simple and purely syntactic, from soundness checking, which formally connects the type system to the desired predicates, allows these proofs to be performed automatically.

Dependent ML (DML) [40, 41] allows ML types to depend upon integers with linear inequality constraints. This limited form of dependent types can be used to automatically prove arithmetic program invariants, including those provable by our integer qualifiers like pos and nonzero. DML's types can also express arithmetic invariants that relate multiple program expressions, which are not supported in our framework.

The technical details of our approach build on our previous work on the Cobalt and Rhodium languages [25, 26]. These languages allow users to implement dataflow analyses that can be automatically proven sound by discharging proof obligations with an automatic theorem prover. Our case and assign rules are similar to Rhodium's syntax for flow functions; our restrict and disallow rules have no analogue in Cobalt or Rhodium. Because our work is based on type systems rather than dataflow analysis, our framework's implementation and formalization are quite distinct from those of Cobalt and Rhodium. Our framework also must handle new issues, including subtyping and the distinction between value and reference qualifiers. On the other hand, Cobalt and Rhodium, being based on dataflow analysis, are naturally flow sensitive.

\section{Conclusions and Future Work}

We have presented a new approach for supporting user-defined type refinements. We allow programmers to supply explicit type rules for new refinements, enabling the expression of common typing disciplines that would be difficult or unnatural to express in prior frameworks. An extensible typechecker executes these rules on programs, and a soundness checker validates the correctness of these rules once, for all possible programs. We have demonstrated the approach through a framework for adding type qualifiers to $\mathrm{C}$ programs. Our framework supports the expression of a variety of qualifiers, and we have used these qualifiers to statically ensure interesting run-time invariants in open-source $\mathrm{C}$ programs.

We plan to explore several directions to increase the expressiveness and practicality of the approach. First, we will incorporate techniques to make existing qualifiers more flexible. We are currently extending the pattern language to support special-purpose behavior for procedure calls and returns, to make use of the semantics of variable scoping. Other extensions include support for qualifier inference to decrease the annotation burden and support for a form of flow sensitivity.

Second, we will extend our framework to handle new kinds of qualifiers. Flow sensitivity will allow us to explore the specification and checking of temporal protocols. We will also target qualifiers, like const, whose associated invariants are naturally expressed as predicates over execution traces. Reasoning automatically about execution traces is a challenge for our soundness checker. We plan to convert trace-based invariants into predicates on a single execution state by allowing users to conservatively instrument a program's dynamic semantics to record extra information [26]. Also, more work is needed to find the right primitives that allow a wide variety of practical reference qualifiers to be specified and proven sound automatically.

Finally, it is possible that our approach can be used beyond type qualifiers, for example to support program checking with semantic guarantees for higher-level programming idioms such as design patterns [21].

\section{Acknowledgments}

This research was supported in part by NSF ITR award \#0427202 and by a generous gift from Microsoft Research. Thanks to Craig Chambers, Jeff Foster, Sorin Lerner, Jens Palsberg, and Ben Titzer for helpful comments on the paper.

\section{References}

[1] J. Aldrich, V. Kostadinov, and C. Chambers. Alias annotations for program understanding. In Proceedings of the 17th ACM SIGPLAN conference on Object-oriented programming, systems, languages, and applications, pages 311-330. ACM Press, 2002.

[2] K. Arnold, J. Gosling, and D. Holmes. The Java Programming Language Third Edition. Addison-Wesley, Reading, MA, third edition, 2000.

[3] C. Bailleux. More security problems in bftpd-1.0.12. bugtraq mailing list post of December 8, 2000. http://www. securityfocus. com/archive/1/149977.

[4] C. Boyapati, R. Lee, and M. Rinard. Ownership types for safe programming: Preventing data races and deadlocks. In Proceedings of the 17th ACM SIGPLAN conference on Object-oriented programming, systems, languages, and applications, pages 211-230. ACM Press, 2002.

[5] J. Boyland. Alias burying: Unique variables without destructive reads. Softw. Pract. Exper., 31(6):533-553, 2001.

[6] C\# Language Specifi cation, Second Edition. ECMA International, Standard ECMA-334, Dec. 2002. 
[7] B. Chin, S. Markstrum, and T. Millstein. Semantic type qualifi ers. Technical Report CSD-TR-40045, UCLA Computer Science Department, November 2004.

[8] D. G. Clarke, J. M. Potter, and J. Noble. Ownership types for fexible alias protection. In Proceedings of the 13th ACM SIGPLAN conference on Object-oriented programming, systems, languages, and applications, pages 48-64. ACM Press, 1998.

[9] R. L. Constable, S. F. Allen, H. M. Bromley, W. R. Cleaveland, J. F. Cremer, R. W. Harper, D. J. Howe, T. B. Knoblock, N. P. Mendler, P. Panangaden, J. T. Sasaki, and S. F. Smith. Implementing Mathematics with the Nuprl Proof Development System. PrenticeHall, Englewood Cliffs, NJ, 1986.

[10] T. Coquand and G. Huet. The calculus of constructions. Inf. Comput., 76(2-3):95-120, 1988.

[11] K. Crary and J. C. Vanderwaart. An expressive, scalable type theory for certifi ed code. In Proceedings of the seventh ACM SIGPLAN international conference on Functional programming, pages 191205. ACM Press, 2002.

[12] R. DeLine and M. Fahndrich. Enforcing high-level protocols in lowlevel software. In Proceedings of the ACM SIGPLAN 2001 conference on Programming language design and implementation, pages 59-69. ACM Press, 2001.

[13] R. DeLine and M. Fahndrich. Typestates for objects. In Proceedings of the 2004 European Conference on Object-Oriented Programming, LNCS 3086, Oslo, Norway, June 2004. Springer-Verlag.

[14] D. Detlefs, G. Nelson, and J. B. Saxe. Simplify: A theorem prover for program checking. Technical Report HPL-2003-148, HP Labs, 2003.

[15] M. Fahndrich and K. R. M. Leino. Declaring and checking non-null types in an object-oriented language. In Proceedings of the 18th ACM SIGPLAN conference on Object-oriented programing, systems, languages, and applications, pages 302-312. ACM Press, 2003.

[16] C. Flanagan and S. N. Freund. Type-based race detection for Java. In Proceedings of the ACM SIGPLAN 2000 conference on Programming language design and implementation, pages 219-232. ACM Press, 2000.

[17] C. Flanagan, K. R. M. Leino, M. Lillibridge, G. Nelson, J. B. Saxe, and R. Stata. Extended static checking for Java. In Proceedings of the ACM SIGPLAN '02 Conference on Programming Language Design and Implementation, June 2002.

[18] C. Flanagan and S. Qadeer. A type and effect system for atomicity. In Proceedings of the ACM SIGPLAN 2003 conference on Programming language design and implementation, pages 338-349. ACM Press, 2003.

[19] J. S. Foster, M. Fähndrich, and A. Aiken. A Theory of Type Qualifi ers. In Proceedings of the 1999 ACM SIGPLAN Conference on Programming Language Design and Implementation, pages 192-203, Atlanta, Georgia, May 1999.

[20] J. S. Foster, T. Terauchi, and A. Aiken. Flow-sensitive type qualifi ers. In Proceedings of the ACM SIGPLAN 2002 Conference on Programming language design and implementation, pages 1-12. ACM Press, 2002.

[21] E. Gamma, R. Helm, R. E. Johnson, and J. Vlissides. Design Patterns: Elements of Reusable Object-Oriented Software. Addison-Wesley, Massachusetts, 1995.

[22] J. Gosling, B. Joy, G. Steele, and G. Bracha. The Java Language Specifi cation Second Edition. The Java Series. Addison-Wesley, Boston, Mass., 2000.

[23] D. Grossman, G. Morrisett, T. Jim, M. Hicks, Y. Wang, and J. Cheney. Region-based memory management in Cyclone. In Proceedings of the ACM SIGPLAN 2002 Conference on Programming language design and implementation, pages 282-293. ACM Press, 2002.

[24] R. Johnson and D. Wagner. Finding user/kernel pointer bugs with type inference. In Proceedings of the 13th USENIX Security Symposium, pages 119-134, 2004.
[25] S. Lerner, T. Millstein, and C. Chambers. Automatically proving the correctness of compiler optimizations. In Proceedings of the ACM SIGPLAN 2003 Conference on Programming Language Design and Implementation, pages 220-231. ACM Press, 2003.

[26] S. Lerner, T. Millstein, E. Rice, and C. Chambers. Automated soundness proofs for datafbw analyses and transformations via local rules. In Proceedings of the 32nd ACM SIGPLAN-SIGACT Symposium on Principles of Programming Languages. ACM Press, 2005.

[27] Y. Mandelbaum, D. Walker, and R. Harper. An effective theory of type refi nements. In Proceedings of the eighth ACM SIGPLAN international conference on Functional programming, pages 213225. ACM Press, 2003.

[28] P. Martin-Löf. Constructive mathematics and computer programming. In Sixth International Congress for Logic, Methodology, and Philosophy of Science, pages 153-175, Amsterdam, 1982. NorthHolland.

[29] R. Milner, M. Tofte, R. Harper, and D. MacQueen. The Defi nition of Standard ML (Revised). The MIT Press, 1997.

[30] G. Morrisett, D. Walker, K. Crary, and N. Glew. From System F to typed assembly language. ACM Trans. Program. Lang. Syst., 21(3):527-568, 1999.

[31] G. C. Necula. Proof-carrying code. In Proceedings of the 24th ACM SIGPLAN-SIGACT Symposium on Principles of Programming Languages, pages 106-119. ACM Press, 1997.

[32] G. C. Necula, S. McPeak, S. P. Rahul, and W. Weimer. CIL: Intermediate Language and Tools for Analysis and Transformation of C Programs. In Proceedings of CC 2002: 11'th International Conference on Compiler Construction. Springer-Verlag, Apr. 2002.

[33] G. C. Necula, S. McPeak, and W. Weimer. CCured: Type-safe retrofi tting of legacy code. In Proceedings of the 29th ACM SIGPLAN-SIGACT Symposium on Principles of Programming Languages, pages 128-139. ACM Press, 2002.

[34] G. Nelson and D. C. Oppen. Simplifi cation by cooperating decision procedures. ACM Trans. Program. Lang. Syst., 1(2):245-257, 1979.

[35] D. Rémy and J. Vouillon. Objective ML: An effective object-oriented extension of ML. Theory and Practice of Object Systems, 4(1):27-52, 1998.

[36] U. Shankar, K. Talwar, J. S. Foster, and D. Wagner. Detecting Format String Vulnerabilities with Type Qualifi ers. In Proceedings of the 10th Usenix Security Symposium, Washington, D.C., Aug. 2001.

[37] Z. Shao, B. Saha, V. Trifonov, and N. Papaspyrou. A type system for certifi ed binaries. In Proceedings of the 29th ACM SIGPLAN-SIGACT Symposium on Principles of Programming Languages, pages 217232. ACM Press, 2002.

[38] M. Tofte and J.-P. Talpin. Implementation of the typed call-by-value $\lambda$-calculus using a stack of regions. In Proceedings of the $21 \mathrm{st}$ ACM SIGPLAN-SIGACT Symposium on Principles of Programming Languages, pages 188-201. ACM Press, 1994.

[39] A. K. Wright and M. Felleisen. A syntactic approach to type soundness. Information and Computation, 115(1):38-94, 15 Nov. 1994.

[40] H. Xi and F. Pfenning. Eliminating array bound checking through dependent types. In Proceedings of ACM SIGPLAN Conference on Programming Language Design and Implementation, pages 249-257, Montreal, June 1998.

[41] H. Xi and F. Pfenning. Dependent types in practical programming. In Proceedings of the 26th ACM SIGPLAN Symposium on Principles of Programming Languages, pages 214-227, San Antonio, January 1999. 